\title{
Maximizing dose reductions with cardiac CT
}

\author{
Matthew J. Budoff
}

Received: 16 October 2008/Accepted: 10 December 2008/Published online: 30 December 2008

(C) The Author(s) 2008. This article is published with open access at Springerlink.com

\begin{abstract}
Multidetector computed tomography has come a long way in a short time, quickly becoming a standard tool in the cardiac imaging armamentarium. The promise of plaque imaging, combined with both anatomical visualization and stenosis detection, has made this a preferred first line test of many cardiologists and radiologists. This test is well suited to rule out coronary artery disease (obstruction) and still diagnosing subclinical plaque, with may be a good target for anti-atherosclerotic therapies. There has been recent criticism against CT imaging, and cardiac CT specifically, due to the high radiation doses that being employed. New advances have allowed for dramatic dose reductions. These include more routinely performed methods such as dose modulation, and newer methods such as prospective gating or minimizing the field of view. This paper will review the different applications to reduce cardiac CT radiation doses to nominal levels, potentially expanding the applications of cardiac CT by removing one of the biggest barriers.
\end{abstract}

Keywords Radiation exposure - Radiation reduction - Millisievert - Noninvasive angiography . CT angiography, CTA $\cdot$ Computed tomographic

M. J. Budoff ( $\square)$

Los Angeles Biomedical Research Institute at Harbor,

UCLA, 1124 W. Carson Street, RB2, Torrance,

CA 90502, USA

e-mail: mbudoff@labiomed.org angiography $\cdot$ Multidetector computed tomography, MDCT $\cdot$ Multislice computed tomography, MSCT

\section{Introduction}

Over the last decade great strides have been made in the field of cardiac imaging, particularly in the ability to view the coronary artery lumen with ample diagnostic accuracy [1]. Current 64-MDCT (multirow detector computed tomography) systems have faster gantry rotation speed resulting in better temporal resolution. Better $Z$-axis spatial resolution is made possible by thin collimations with extensive volumetric acquisitions [2, 3]. The technique of retrospective image acquisition scanning with overlapping of slices that compensates for potential cardiac motion data at all phases of the cardiac cycle to assess ejection fraction valves and wall motion. [2, 3]. This results in more patient radiation exposure in comparison with CTA with prospective-gated acquisition (originally used with the electron beam tomography scanner) [4]. Studies estimate radiation exposure for 16-row at $8.8 \mathrm{mSv}$ for a $16 \times 0.75 \mathrm{~mm}$ scan protocol with a pitch of 0.28 and power of $370 \mathrm{~mA}$ [5], and 13 and $18 \mathrm{mSv}$ (for men and women, respectively) with 64-row MDCT [6]. In a recent study, Pugliese et al. [7] reported radiation doses in the order of 15:20 mSv (male:female), 
respectively. These doses for retrospective imaging are significantly higher than those seen with invasive diagnostic coronary angiography where the dose is between 2.1 and $7 \mathrm{mSv}$ [8,9], but lower than the effective radiation dose for a technetium Tc $99 \mathrm{~m}$ sestamibi myocardial perfusion stress test (9-18 mSv, increasing to over $20 \mathrm{mSv}$ with dual-isotope scanning) $[10,11]$.

\section{Methods to minimize dose}

Multiple different radiation reductions methods have been reported, all of which can result in complementary reductions in radiation exposure. This paper outlines reported and potential methods, as well as discusses the potential for future radiation reduction techniques. While it is logical and in keeping with the principles of ALARA (as low as reasonably achievable) to use the minimum radiation dose necessary in cardiac CT examinations, there are little data testing the effect of specific radiation sparing approaches on diagnostic quality. Cardiac CTA imaging protocols are also not as widely standardized, and thus maintaining diagnostic accuracy is important. An examination which is non-diagnostic, of course, exposes the patient to radiation without benefit. However, experience dictates that through attention to detail of the CT acquisition, excellent image quality can be maintained at a lower radiation exposure.

\section{Dose related issues}

\section{Limiting craniocaudal coverage length}

The region of the patient's body that undergoes radiation exposure in the craniocaudal, or z-direction is the largest contributor to radiation absorption (via changes in the dose length product [DLP]). For cardiac CT examinations, proper attention to scan length should be made. For example, rather than rigidly scanning from carina to diaphragm as is frequently recommended by scanner manufacturers, the operator could potentially use the non-contrast images for calcium scoring, if necessary to ascertain the most cranial and caudal position of the coronary arteries.

Using the scout image, the field of view usually extends from $\sim 1 \mathrm{~cm}$ below the carina to just below the diaphragm to ensure complete coronary imaging (Fig. 1). The scout film is highly inaccurate for determining the precise origin of the coronary arteries [12]. An alternative is to use images from the coronary calcium scoring to set the upper limit above the apex of the left anterior descending artery (the most superior artery) and the lower limit inferior to the posterior descending artery, leaving sufficient but not excessive margins, to allow for movement. Calcium scoring with MDCT delivers $\sim 1.2 \mathrm{mSv}$ when acquired with prospective ECG gating, and potentially lower if $100 \mathrm{kVp}$ is used for this acquisition [4]. A

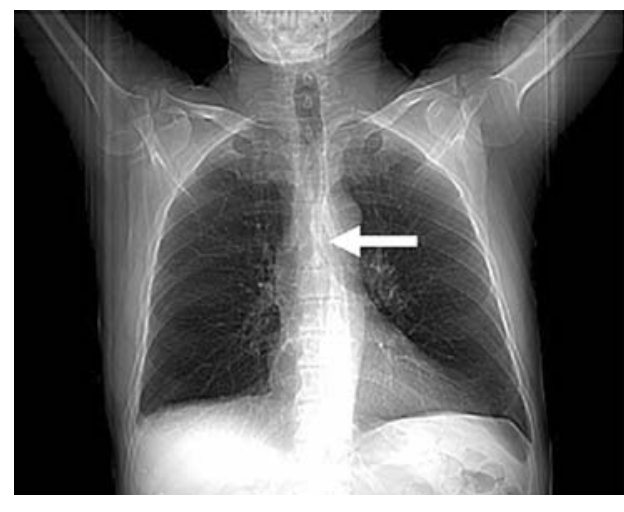

Fig. 1 A scout film (left panel) used to identify the upper and the lower limits of the region of interest for the subsequent CTA. The white arrow shows the level of the carina, which is a landmark for determining the top of the coronary tree. Middle panel An axial slice of a non-contrast coronary calcium scan showing the left main artery (black arrow). The exact level in which the superior most coronary arteries arise is most
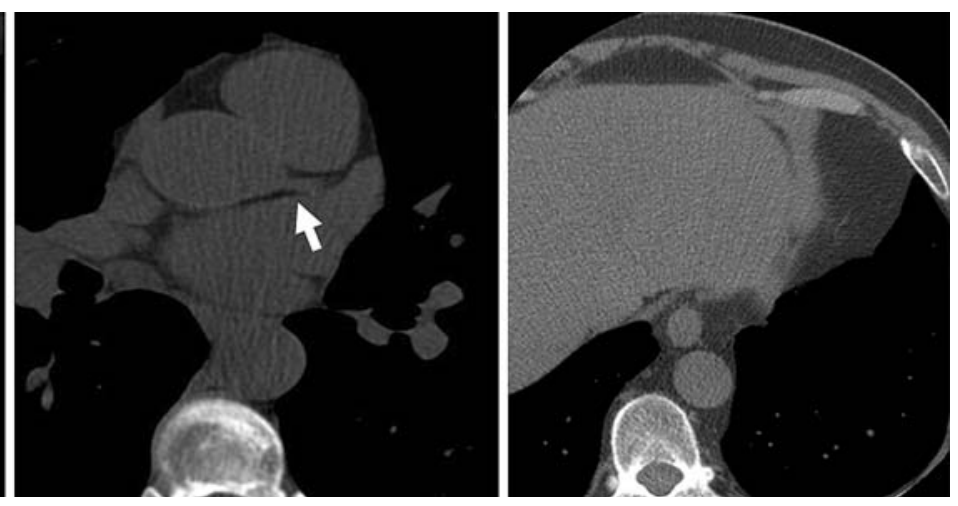

effectively visualized with axial data such as the calcium scan. Right panel An axial slice of a non-contrast coronary calcium scan defining the lower limits of the heart, demonstrating the level just below the posterior descending artery. There are no coronary arteries on this image, so concluding the CTA just below this would safely allow for complete imaging of the coronary tree 
$30 \%$ reduction in radiation dose has been shown by employing a calcium score to determine the superior and interior borders of the field of view, with the superior border at $1 \mathrm{~cm}$ above the visualized top of the coronary arteries and the inferior border at $1 \mathrm{~cm}$ below the posterior descending artery [13]. The savings in radiation dose was $4 \mathrm{mSv}$ offsetting the dose delivered by calcium scoring $(1.2 \mathrm{mSv})$. The average percentage reduction in radiation was $30 \%$ $(\mathrm{SD} \pm 10)$ with a median of $22 \%$. This method of dose reduction is incremental to dose modulation as well as reducing the $\mathrm{mA}$ or $\mathrm{kV}$ during image acquisition. Multipurpose examinations of the chest have long $Z$ axis acquisitions and thus higher radiation dose. Multipurpose examinations of the chest tend to have long $Z$ axis acquisitions and very high radiation exposures. For example, to do a "triple rule out" examination, both scan length and scan time are affected, and there is a consequent marked increase in radiation exposure. Thus, this protocol should be clinically indicated to justify the additional radiation.

\section{Tube amperage}

Tube $\mathrm{mA}$ is a variable setting that affects the number of photons generated and directly affects image signal to noise ratio. Radiation dose is approximately proportional to $\mathrm{mA}$, and customarily, amperage can be adjusted for body mass and configuration [3]. Patients with higher mass will experience higher photon scatter and higher noise, while thinner patient will have images of good quality using lower amperage. Failure to adjust $\mathrm{mA}$ downward for thin patients will result in unnecessary radiation. It is important to reduce $\mathrm{mA}$ to the lowest necessary to make diagnostic images. Typical doses are $350 \mathrm{~mA}$ for small patients, $450 \mathrm{~mA}$ for medium sized patients and 550 or higher for large patients (scaled to maximum $\mathrm{mA}$ of the $\mathrm{X}$-ray tube for the heaviest patients). Typical settings from the manufacturers are pre-set higher, to optimize image quality at the expense of radiation dose to the patient.

Dose modulation (electrocardiographic pulsing)

Current generation scanners are capable of varying tube current output (mA) in synchrony to the patient's electrocardiogram. This is done to reduce radiation during phases in the cardiac cycle when the heart is moving more dynamically (ventricular systole and end diastole [which correlates with atrial systole]). The ideal "pulsing window" (when current becomes maximal) is as short as possible, typically focused around the $70 \%$ phase of the cardiac cycle. This becomes a complex decision as there is a trade-off between pulse window width, heart rate and scanner type. All current-generation cardiac-capable scanners have built-in software that either varies pulsing window width with heart rate or allows the operator to customize these protocols. The use of ECG pulsing can decrease radiation dose by $50 \%$ or more and is generally recommended unless other parameters threaten the image quality (such as irregular heart rate). The time of least motion occurs between 40 and $80 \%$ of the R-R interval (mid diastole) $[14,15]$. With dose modulation, the dose is reduced by $18-47 \%$ depending on the patient's heart rate [16-18]. Slower heart rates will lead to more effective use of dosemodulation and lower radiation doses (with the exception of the dual source scanner, where slower heart rates will increase radiation exposure). The multicenter 64-MDCT ACCURACY trial utilized dose modulation and still reported negative predictive values of $99 \%$ [1]. While EKG-regulated dose modulation could be implemented in a majority of patients, sometimes it cannot be utilized due to scanning conditions requiring additional image reconstructions during different phases of the cardiac cycle (such as irregular heart rhythms and fast heart beats). Although the radiation burden of cardiac CT studies can be efficiently reduced by dose modulation, a further decrease in radiation without compromising diagnostic image quality would be indeed very desirable [19].

\section{Scanner type}

In general, increasing the number of detector-rows and reducing detector size tends to increase the radiation dose due to the increasing surface area of lead collimators (which can only be so thin while still being effective) in comparison to detector area. The more lateral detectors that are present lead to less efficient delivery of dose, as the lateral detectors (those not directly across from the X-ray tube) require more radiation exposure due to scatter. Thus, four row scanners had lower patient radiation doses than 16 slice scanners, despite longer radiation times. 
Similarly 16 row scanners impart lower doses than 64, and subsequently, doses from 64 will be lower than 256 and 320 row scanners (for similar protocols). In addition, complex effects are also produced by dual source scanners which have two X-ray sources and detector rings operating during the scan time, but have a reduced scan time and heart-rate variable pitch. Theoretically, dual source scanners reduce the scan duration because their faster temporal resolution allows imaging faster heart rates thereby reducing diastolic time, which balances the double dose of radiation being emitted, leading to similar doses as 64 row MDCT scanners and lower doses than 256 and 320 row scanners.

Reducing tube voltage

Another methodology to reduce the dose is to reduce tube voltage. Tube $\mathrm{kVp}$ affects the peak photon energy and affects image contrast. In general, $\mathrm{kVp}$ has been used to frequently adjust for body mass. New studies suggest that that protocols utilizing 100 or $80 \mathrm{kVp}$ may be effective in thin patients for reducing dosage in coronary calcium measurements or coronary CT angiography, without degrading diagnostic accuracy [20]. Tube voltage has a more dramatic effect on radiation dosage, which varies approximately with the square of the $\mathrm{kVp}$. Budoff et al. [21] demonstrated use of $100 \mathrm{kVp}$ reduced radiation dose $42 \%$ using prospective triggering and $40 \%$ using retrospective imaging, as compared to $120 \mathrm{kVp}(P<0.001)$. Hausleiter et al. [22] showed a $53-64 \%$ reduction in estimated radiation dose using both reduced $\mathrm{kVp}$ and dose modulation. As noted above, this benefit is independent and incremental to dose modulation radiation savings and other savings listed above (Table 1).
Limiting field of view (Fov)

An important technique that is currently underused, which will both minimize radiation and afford better image quality, is to restrict the $x y$ field of view. A smaller $x y$ field of view will improve image quality, as the FOV divided by 512 is the resolution in the $X-Y$ axis (to an optimal resolution of about $0.3-0.35 \mathrm{~mm}$ for current scanners). The bowtie filter allows for smaller radiation exposure by limiting the scatter of the X-ray towards the detectors. Intrinsically, it is more efficient to irradiate detectors directly across from the X-ray tube and thus, requires less photons. Thus, the bowtie filter allows less X-rays out of the central part of the X-ray tube, as less are required to expose the detectors. As one moves laterally from the center of the detector array, the number of photons needed to create an image is higher (less efficiency and thus, the relative dose increases for scanners with higher numbers of detectors (see \#4 above). The bowtie filter allows more photons to go toward the lateral detectors, thus looking like a bowtie, with a small center and large lateral edges. These filters are optimized for patient size, and the smallest bowtie filters, only allow the lateral tissue to be exposed to $32 \mathrm{~cm}$, the medium bowtie to $36 \mathrm{~cm}$ and the largest bowtie to over $40 \mathrm{~cm}$. Since all hearts (including bypass patients) fit in a small bowtie filter, the use of larger bow-ties would only be necessary when larger field of views are needed. In a study of radiation doses (measured radiation exposures using the LightSpeed VCT 64-MDCT scanner (GE Healthcare, Waukesha, WI, USA), the dose using the small cardiac bowtie (can only be post-processed up to $32 \mathrm{~cm}$ field of view), resulted in a dose of $8 \mathrm{mSv}$ (retrospective imaging, as low as $1 \mathrm{mSv}$ with prospective imaging) [23]. A medium bowtie filter for a retrospectively acquired

Table 1 Expected dose reductions based upon different scanning strategies

\begin{tabular}{llll}
\hline Dose reduction technique & $\begin{array}{l}\text { Effectively lowers exposure } \\
\text { with retrospective gating }\end{array}$ & $\begin{array}{l}\text { Estimated dose reduction } \\
\text { for retrospective }\end{array}$ & $\begin{array}{l}\text { Effectively lowers exposure } \\
\text { with prospective triggering }\end{array}$ \\
\hline Scan length & $\downarrow \downarrow \downarrow$ & Proportional & $\downarrow$ \\
Adding calcium Score to lower scan length & $\downarrow \downarrow$ & $30 \%$ & $\uparrow$ \\
Reducing amperage (mA) & $\downarrow$ & Proportional & $\downarrow$ \\
Dose modulation & $\downarrow \downarrow \downarrow$ & $20-40 \%$ & Not applicable \\
Reducing tube voltage to $100 \mathrm{kVp}$ & $\downarrow \downarrow \downarrow$ & $40 \%$ & $\downarrow \downarrow \downarrow$ \\
Limiting scan field of view & $\downarrow \downarrow \downarrow$ & $67 \%$ & $\downarrow \downarrow$ \\
\hline
\end{tabular}

$\downarrow$-Mild decrease; $\downarrow \downarrow$-moderate decrease; $\downarrow \downarrow \downarrow$-large decrease in radiation dose 
cardiac CT angiogram using the same protocol affords $13.4 \mathrm{mSv}$ of radiation. This is a $40 \%$ dose reduction by simply using an appropriate sized bowtie filter for the study required (cardiac CT requires at most $25 \mathrm{~cm}$ of view). Larger patients may require a medium bowtie filter due to optimization protocols, but for cardiac applications, a large bowtie filter would never be necessary. The routine use of small bowtie filters (and small FOV) will both reduce radiation dose and limit incidental findings related to non-cardiac disease (primarily non-cancerous lung nodules). There is no evidence yet to support that discovering these incidental findings will lead to improvement in outcomes. There is data available that these incidental findings increase surgical rates, costs and patient anxiety [24]. For recommended bowtie filters based on body habitus for the GE VCT Scanner, see Table 2. These findings need to be validated for other scanners and manufacturers.

Sequential (prospective) scanning versus retrospective gating

Until recently, all studies were done with retrospective gating, using continuous $\mathrm{X}$-ray beam exposure to create thousands of images, then selecting a subset of those at the optimal phase of the cardiac cycle for interpretation. An alternative imaging mode is the axial step-and-shoot acquisition (prospective gating). This was first used with electron beam CT angiography as early as 1995, and with calcium scoring with MDCT since 1998. Approximately 2 years ago, prospective gating was introduced for MDCT angiography. This is by far the most significant dose reduction technique, as it turns off the X-ray tube except for a short exposure period centered around the ideal imaging phase (typically mid-diastole). In patients with slow and very steady heart rates, extremely low radiation doses can be achieved by sequential scanning with tube output only during a narrow ECG window (Table 3). The X-ray beam is triggered by the ECG and is turned on only during the required phase of the cardiac cycle (usually $75 \%$ of the cardiac cycle) and turned off during the rest of the cardiac cycle. Retrospective ECG gating can be done with or without 'padding'. Padding provides additional phase information to account for variations in heart rate by adding time before and after the center phase of the acquisition (e.g. adding $100 \mathrm{~ms}$ before and after the $75 \%$ phase thus providing additional phase information). Using the GE technology, padding is prescribed in the range of $0-200 \mathrm{~ms}$ and is added to both sides of the center of the acquisition. This allows more phases of data to be available to the reader and is generally used when heart rates are $>60 \mathrm{bpm}$ or when heart rate variability is noted. The padding should be wider with more rapid heart rates [25]. When padding was used, it is added in a heart rate dependent fashion as follows: 30-39 bpm, $175 \mathrm{~ms}$ padding; 40-49 bpm, $150 \mathrm{~ms}$ padding; 50$59 \mathrm{bpm}, 125 \mathrm{~ms}$ padding and $\geq 60 \mathrm{bpm}, 100 \mathrm{~ms}$ padding. Padding default is set to approximately $\pm 10 \%$ of the patient's R-R cycle. Patients with very stable and slow heart rates underwent no padding to

Table 2 Radiation doses of retrospective imaging with 64 MDCT based on body mass index (BMI)

\begin{tabular}{|c|c|c|c|c|c|c|c|c|c|}
\hline \multirow[t]{2}{*}{ BMI } & & \multicolumn{2}{|c|}{ Calcium scan } & \multicolumn{2}{|c|}{ Retrospective } & \multirow[t]{2}{*}{$\mathrm{KvP}$} & & \multirow[t]{2}{*}{ DLP } & \multirow{2}{*}{$\begin{array}{l}\text { Estimated } \\
\text { mSv including } \\
\text { CAC scan }\end{array}$} \\
\hline & & $\mathrm{kVp}$ & $\mathrm{mA}$ & Max mA & Min $\mathrm{mA}$ & & & & \\
\hline 19 and less & 250 & 120 & 300 & 280 & 140 & 100 & C2/Small filte & 244 & 4.148 \\
\hline $20-22$ & 275 & 120 & 350 & 325 & 170 & 100 & C2/Small filte & 289 & 4.913 \\
\hline $23-25$ & 300 & 120 & 375 & 350 & 190 & 100 & C2/Small filte & 311 & 5.287 \\
\hline $26-28$ & 325 & 120 & 450 & 425 & 215 & 100 & C2/Small filte & 359 & 6.103 \\
\hline $29-31$ & 350 & 120 & 500 & 475 & 240 & 120 & C2/Small filte & 640 & 10.88 \\
\hline $32-35$ & 375 & 120 & 600 & 575 & 290 & 120 & C2/Small filte & 772 & 13.124 \\
\hline $36-37$ & 400 & 120 & 700 & 650 & 290 & 120 & C3/Med filter & 954 & 16.218 \\
\hline$\geq 38$ & 425 & 120 & 800 & 750 & 375 & 120 & C3/Med filter & 1,170 & 19.89 \\
\hline$>40$ & 450 & 120 & 800 & 775 & 388 & 120 & C3/Med filter & 1,270 & 21.59 \\
\hline
\end{tabular}

$D L P$ dose length product; $k V p$ killivolts; $m A$ milliamperes; $C A C$ coronary artery calcium scan 
Table 3 SnapShot Pulse (prospective acquisition) GE-VCT parameters

\begin{tabular}{|c|c|}
\hline Action & Parameter \\
\hline Cardiac mode & $\begin{array}{l}\text { Prospective step-and-shoot (SnapShot } \\
\text { Pulse) }\end{array}$ \\
\hline Rotation time & $0.35 \mathrm{~s}$ \\
\hline $\begin{array}{l}\text { Start location and } \\
\text { end location }\end{array}$ & $\begin{array}{l}\text { Based on scout coverage-base of the } \\
\text { heart to apex (about } 1 \mathrm{~cm} \text { above the } \\
\text { left main artery to about } 1 \mathrm{~cm} \text { below } \\
\text { the posterior descending artery) }\end{array}$ \\
\hline Detector coverage & $40 \mathrm{~mm}$ \\
\hline Slice thickness & $0.625 \mathrm{~mm}$ \\
\hline Padding & Set by system or turned off \\
\hline $\begin{array}{l}\text { SFOV (scan field } \\
\text { of view) }\end{array}$ & $\begin{array}{l}\text { Small or medium depending on the body } \\
\text { habitus of the patient }\end{array}$ \\
\hline DFOV & $\begin{array}{l}\text { Typically } 18 \text { or } 15 \mathrm{~cm} \text { field of view to } \\
\text { include all of the cardiac structures }\end{array}$ \\
\hline \multirow[t]{2}{*}{$\mathrm{kVp}$} & $\begin{array}{l}120 \mathrm{kVp} \geq 85 \mathrm{~kg} \text { patient (obese or } \\
\text { morbidly obese) }\end{array}$ \\
\hline & $100 \mathrm{kVp}$ for $<85 \mathrm{~kg}$ patient \\
\hline \multirow[t]{3}{*}{$\mathrm{mA}$} & 350 - Small patient \\
\hline & 450-Medium patient \\
\hline & $550+$-Large patient \\
\hline
\end{tabular}

Adapted from Gopal et al. [13]

ensure the lowest possible radiation dose. The prescribed PA window is defined as center phase \pm milliseconds padding (Fig. 2).

Multiple studies have demonstrated similar or superior image quality with prospective imaging (LightSpeed VCT 64-MDCT scanner), and resulted in average doses of 1-3 mSv [26-28]. Superior image quality with prospective imaging is most likely due to lack of ' $Z$ axis' motion, as there is no movement of the table (zero pitch) during image acquisition. Multiple studies have reported mean patient radiation doses 77-80\% lower for prospective gating than for retrospective gating, without compromising image quality or diagnostic accuracy. Budoff et al. [21] showed a 90\% reduction in estimated radiation dose using a combination of reduced $\mathrm{kVp}(100)$ and prospective triggering together. In a majority of patients, patients can undergo prospective triggering with marked radiation dose reduction, if ejection fraction information or multiple phase acquisition is not necessary. This removes one of the biggest barriers to more widespread implementation of cardiac CT [29].

\section{Future horizons for dose reduction}

The newly released High Definition CT (CT750 HD, GE Healthcare, Waukesha, WI, USA) uses the first new detector material in 20 years. This detector material, by changing the molecular structure of real garnets, a scintillator that is proposed to deliver images 100 times faster, with up to $33 \%$ greater detail through the body and up to $47 \%$ greater detail in the heart. This also allows up to 2,496 views per rotation (a $2.5 \times$ increase) to deliver improved spatial resolution and improved image quality across the entire field of view. While validation studies are now underway, the vendor reports a reduced dose by as much as $83 \%$ for cardiac CT. The exact application
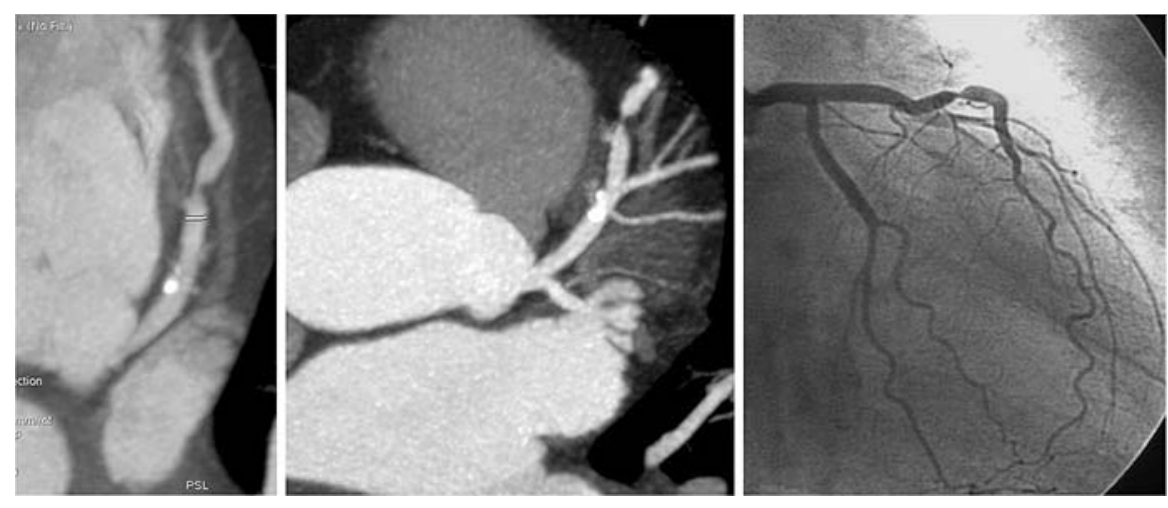

Fig. 2 Shows stenosis in left anterior descending from patient who underwent both cardiac gated helical (RS-OHA) and SnapShot Pulse acquisitions (PA) within 1 week. Images courtesy of Cardiovascular Medical Group, Beverly Hills,
CA. Left panel is retrospective image, middle panel is prospective image and right panel is corresponding cardiac catheterization 


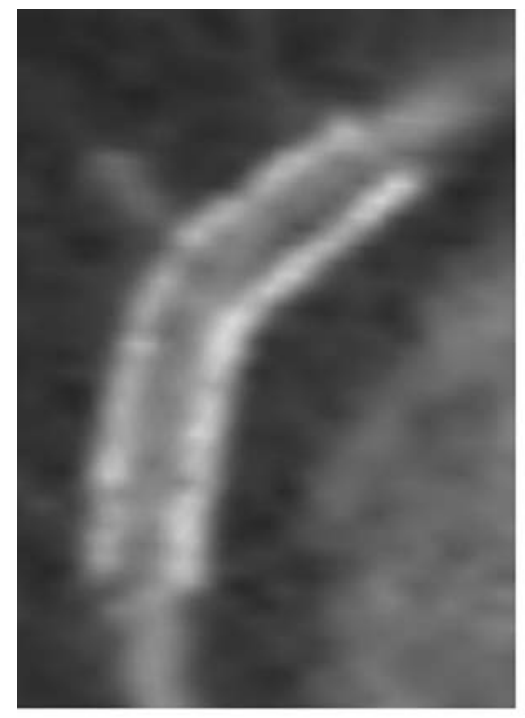

64-MDCT
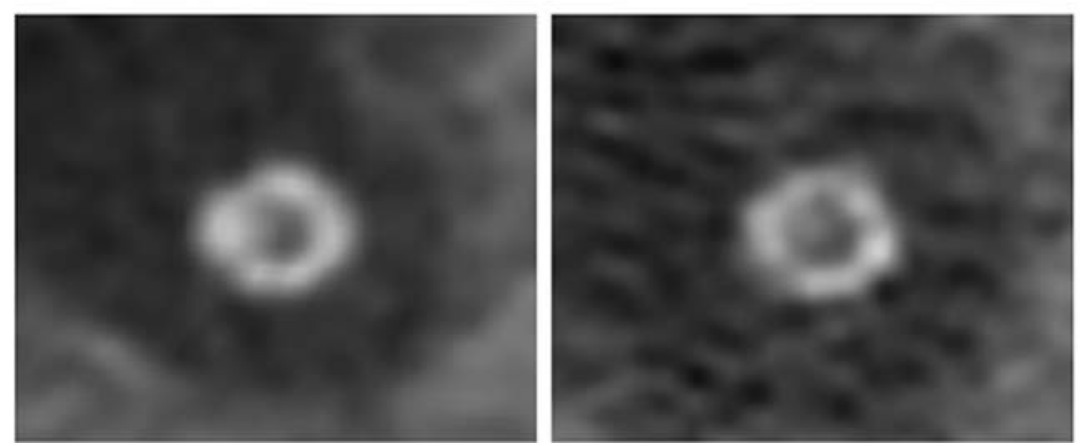

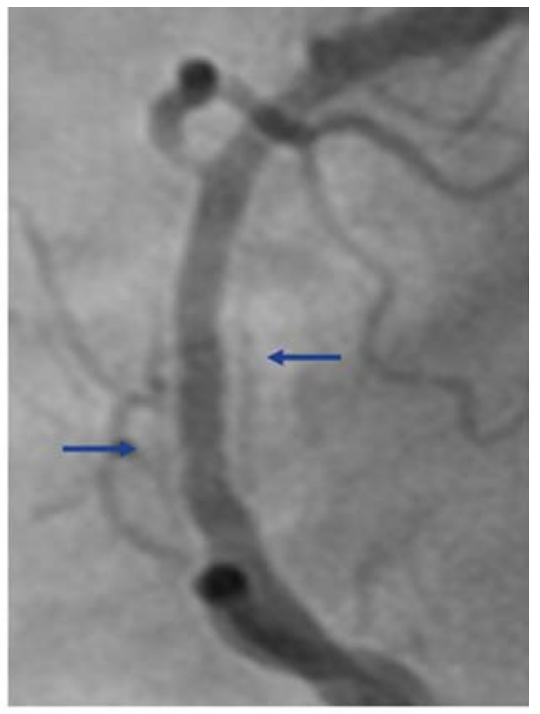

High Def CT

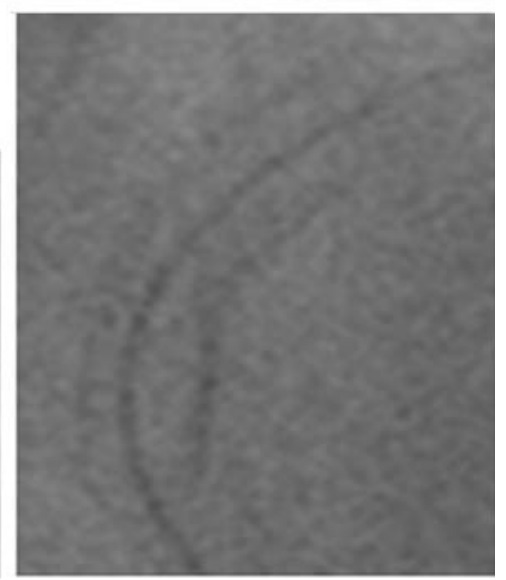

Fig. 3 Left image Resolution of stent impaired with current 64-multi-detector computed tomography. Middle image Improved visualization of in-stent restenosis by High Definition CT, with confirmation of in-stent restenosis by invasive

and overlap with other dose reduction techniques remains to be studied, but the use of more efficient detectors may reduce signal to noise ratios without requiring the typical increase in radiation dose. The High Definition CT should improve visualization of stents and other small structures currently limited by spatial resolution in current 64-320 scanners (Fig. 3).

\section{Summary}

Cardiac CT imaging protocols should be constructed to minimize the radiation exposure to the patient angiography. The arrows represent the stent borders on angiography. Images courtesy of Dr. J. L. Sablayrolles, Centre Cardiologique du Nord, Saint Denis, France

while providing the best possible information for an accurate diagnosis. Diagnostic imaging most likely saves thousands of lives each year by providing medical information to the physician for better medical management of the patient for the overall outcome of the patient's health. While there may be a finite risk associated with a diagnostic study using low level ionizing radiation, the typical patient presents a higher risk by not having the imaging study performed. Using the techniques outlined for adequate patients (based upon body habitus, heart rate and cardiac rhythm), dose reductions up to 70-90\% can be achieved when compared to earlier acquisition strategies for cardiac CT. 
Open Access This article is distributed under the terms of the Creative Commons Attribution Noncommercial License which permits any noncommercial use, distribution, and reproduction in any medium, provided the original author(s) and source are credited.

\section{References}

1. Budoff MJ, Dowe D, Jollis JG, Gitter M, Sutherland J, Halamert E, Scherer M, Bellinger R, Martin A, Benton R, Delago A, Min JK (2008) Diagnostic performance of 64detector row coronary computed tomographic angiography of individuals undergoing invasive coronary prospective multicenter ACCURACY (assessment by coronary computed individuals without known coronary artery disease: results from the tomographic angiography for evaluation of coronary artery stenosis in angiography) trial. J Am Coll Cardiol 52(21):1724-1732

2. Cademartiri F, Malagutti P, Belgrano M et al (2005) Noninvasive coronary angiography with 64-slice computed tomography. Minerva Cardioangiol 53(5):465-472

3. Budoff MF, Gopal A, Gopalakrishnan D (2006) Cardiac computed tomography: diagnostic utility and integration in clinical practice. Clin Card 29(9 Suppl 1):I4-I14

4. Morin RL, Gerber TC, McCollough CH (2003) Radiation dose in computed tomography of the heart. Circulation 107(6):917-922

5. Bae KT, Hong C, Whiting BR (2004) Radiation dose in multidetector row computed tomography cardiac imaging. J Magn Reson Imaging 19:859-863

6. Raff GL, Gallagher MJ, O’Neill WW, Goldstein JA (2005) Spiral computed tomography diagnostic accuracy of noninvasive coronary angiography using 64-slice. J Am Coll Cardiol 46:552-557

7. Pugliese F, Mollet NR, Runza G et al (2006) Diagnostic accuracy of non-invasive 64-slice CT coronary angiography in patients with stable angina pectoris. Eur Radiol 16(3):575-582

8. Mollet NR, Cademartiri F, van Mieghem CA, Runza G, McFadden EP, Baks T, Serruys PW, Krestin GP, de Feyter PJ (2005) High-resolution spiral computed tomography coronary angiography in patients referred for diagnostic conventional coronary angiography. Circulation 112:23182323

9. Kocinaj D, Cioppa A, Ambrosini G, Tesorio T, Salemme L, Sorropago G, Rubino P, Picano E (2006) Radiation dose exposure during cardiac and peripheral arteries catheterisation. Int J Cardiol 113(2):283-284

10. Thompson $\mathrm{R}$, Cullom $\mathrm{S}$ (2006) Issues regarding radiation dosage of cardiac nuclear and radiography procedures. $\mathrm{J}$ Nucl Cardiol 13:19-23

11. Clarke EA, Notghi A, Harding LK (1997) Are MIBI/tetrofosmin heart studies a potential radiation hazard to technologists? Nucl Med Commun 18:574-577

12. Bakhsheshi H, Mao SS, Budoff MJ, Lu B, Brundage BH (2000) Preview method for electron beam scanning of the coronary arteries. Acad Radiol 7:620-626

13. Gopal A, Budoff MJ (2007) A new method to reduce radiation exposure during multi-row detector cardiac computed tomographic angiography. Int $\mathrm{J}$ Cardiol. doi: 10.1016/j.ijcard.2007.08.072

14. Lu B, Mao SS, Zhuang N et al (2001) Coronary artery motion during the cardiac cycle and optimal ECG triggering for coronary artery imaging. Invest Radiol 36(5):250-256

15. Mao S, Lu B, Oudiz RJ et al (2000) Coronary artery motion in electron beam tomography. J Comput Assist Tomogr 24(2):253-258

16. Flohr TG, Schoepf UJ, Kuettner A et al (2003) Advances in cardiac imaging with 16 -section CT systems. Acad Radiol 10(4):386-401

17. Jakobs TF, Becker CR, Ohnesorge B et al (2002) Multislice helical CT of the heart with retrospective EKG gating: reduction of radiation exposure by EKG-controlled tube current modulation. Eur Radiol 12(5):1081-1086

18. Abada HT, Larchez C, Daoud B, Sigal-Cinqualbre A, Paul JF (2006) MDCT of the coronary arteries: feasibility of low-dose CT with EKG-pulsed tube current modulation to reduce radiation dose. AJR Am J Roentgenol 186(6 Suppl 2):S387-S390

19. Budoff MJ (2008) Cardiac CT principles. Chapter 1. In: Budoff MJ, Shinbane J (eds) Handbook of cardiovascular CT, essentials for clinical practice, 1st edn. Springer, London

20. Gopal A, Mao SS, Karlsberg D, Young E, Waggoner J, Ahmadi N, Pal RS, Leal J, Karlsberg RP, Budoff MJ (2008) Radiation reduction with prospective ECG-triggering acquisition using 64-multidetector computed tomographic angiography. Int J Cardiovasc Imaging. doi: 10.1007/s10554-008-9396-z

21. Budoff MJ, Waggoner J, Ahmadi N, Pal RS, Sarlak B, Honoris L, Carson S, Child J, Mao SS, Gopalakrishnan D, Gopal A (2008) Substantial radiation dose reduction in 64multidetector cardiac computed tomographic angiography by using lower X-ray energy during scanning. J Am Coll Cardiol 51:A148

22. Hausleiter J, Meyer T, Hadamitzky M, Huber E, Zankl M, Martinoff S, Kastrati A, Schomig A (2006) Radiation dose estimates from cardiac multislice computed tomography in daily practice. Circulation 113:1305-1310

23. Budoff MJ (2008) Ethical issues related to lung nodules on cardiac CT. Am J Roentg

24. Budoff MJ, Gopal A (2007) Incidental findings on cardiac computed tomography. Should we look? J Cardiovasc Comput Tomogr 1:97-105

25. Steigner ML, Otero HJ, Cai T, Mitsouras D, Nallamshetty L, Whitmore AG et al (2008) Narrowing the phase window width in prospectively ECG-gated single heart beat 320detector row coronary CT angiography. Int J Cardiovasc Imaging. 25(1):85-90. doi:10.1007/s10554-008-9347-8

26. Shuman WP, Branch KR, May JM, Mitsumori LM, Lockhart DW, Dubinsky TJ, Warren BH, Caldwell JH (2008) Prospective versus retrospective ECG gating for 64detector CT of the coronary arteries: comparison of image quality and patient radiation dose. Radiology 248(2):431437

27. Hirai N, Horiguchi J, Fujioka C, Kiguchi M, Yamamoto H, Matsuura N, Kitagawa T, Teragawa H, Kohno N, Ito K (2008) Prospective versus retrospective ECG-gated 64detector coronary CT angiography: assessment of image quality, stenosis, and radiation dose. Radiology 248(2): $424-430$ 
28. Earls JP, Berman EL, Urban BA, Curry CA, Lane JL, Jennings RS, McCulloch CC, Hsieh J, Londt JH (2008) Prospectively gated transverse coronary CT angiography versus retrospectively gated helical technique: improved image quality and reduced radiation dose. Radiology 246(3):742-753
29. Kalra MK, Brady TJ (2008) Current status and future directions in technical developments of cardiac computed tomography. J Cardiovasc Comput Tomogr 2:71-80 